\title{
Ocorrência do pólen de Podocarpus sp. (Podocarpaceae) nas coletas de Frieseomelitta varia Lepeletier 1836 (Apidae: Meliponinae) em uma área de Manaus, AM, Brasil
}

\author{
Antonio Carlos Marques-Souza ${ }^{1,2}$
}

Recebido em 3/11/2009. Aceito em 6/04/2010

RESUMO - (Ocorrência do pólen de Podocarpus sp. (Podocarpaceae) nas coletas de Frieseomelitta varia Lepeletier 1836 (Apidae: Meliponinae) em uma área de Manaus, AM, Brasil). Durante o período de doze meses, o pólen transportado por Frieseomelitta varia Lepeletier 1836 foi coletado das corbículas das operárias, logo após o fechamento da entrada das colméias. Feita a identificação polínica dos grãos de pólen e sua frequência mensal nas amostras, constatou-se que as espécies das famílias mais visitadas pela ordem de coleta, foram: Cecropiaceae, Sapotaceae, Myrtaceae e Moraceae. As operárias coletaram o pólen de 79 espécies de plantas distribuídas em 60 gêneros e 37 famílias, sendo as mais frequentes: Cecropia sp. visitada o ano todo com atratividade em janeiro de 82,03\%, Morus sp. com atratividade de 37,46\% em dezembro; Myrcia amazonica DC. com atratividade em abril de 32,34\% e Pouteria macrophylla (A.DC.) Eyma em junho com atratividade de 36,54\%. Quanto a Podocarpus sp. esse é o primeiro relato da presença do pólen dessa espécie em coletas de meliponíneos, o que não deixa de ser um fato curioso, uma vez que se trata de uma gimnosperma encontrada em áreas específicas da região amazônica e que apresentou atratividade para Frieseomelitta varia Lepeletier 1836 de 4,94\% no mês de março.

Palavras-chave: Abelha sem ferrão, atratividade, nicho trófico, pólen coletado

ABSTRACT - (Occurrence of Podocarpus sp. (Podocarpaceae) pollen on Frieseomelitta varia Lepeletier 1836 (Apidae: Meliponinae) in Manaus, Amazonas, Brazil). Over a 12 month period, pollen was sampled from the corbiculae of workers of the stingless bee Frieseomelitta varia Lepeletier 1836. Collections were obtained immediately after sealing the hives, located in a forest fragment in the city of Manaus. Pollen was identified and monthly frequency of each type was tallied. The most-visited plant families in decreasing order of frequency were Cecropiaceae, Sapotaceae, Myrtaceae and Moraceae. Identified pollen was harvested from 79 plant species in 60 genera and 37 families. The most frequent species were: Cecropia sp., visited year-round but with highest attractiveness (82\%) in January; Morus sp., with peak attractiveness (37\%) in December; Myrcia amazonica DC. with 32\% peak attractiveness in April; and Pouteria macrophylla (A.DC.) Eyma, with 37\% peak attractiveness in June. Curiously, we found pollen of the gymnosperm Podocarpus, which is quite rare in Amazonia, known from only a few sites. As far as we know, this is the first report of Podocarpus pollen on Meliponinae bees. For these urban hives of Frieseomelitta varia Lepeletier, Podocarpus had a peak attractiveness of $4.94 \%$ in the month of March.

Key words: Attractiveness, pollen collected, stingless bees, trophic niche

\section{Introdução}

Na procura pelo alimento os meliponíneos não dispensam qualquer fonte alimentar que possa lhes trazer vantagens e por isso diversificam a sua dieta coletando o pólen e/ou néctar de plantas não correlacionadas entre si (KleinertGiovannini \& Imperatriz-Fonseca 1987; Marques-Souza et al. 2002; Ramalho 1990). As estratégias de coletas dos meliponíneos, e de serem generalistas na procura pelo alimento em fontes diversas, são características básicas de abelhas eussociais (Carvalho et al. 2001; Kerr et al. 1986/87; Michener 1979; Ramalho et al. 1994). Porém, os meliponíneos podem realizar especializações temporárias quando o recurso floral é muito atrativo e apresenta facilidades em sua coleta, fato observado em plantas com grandes floradas (Guibu et al. 1988; Imperatriz-Fonseca et al. 1989; Marques-Souza 1996; Ramalho et al. 1998; 2007).

De outro modo, abelhas sociais diversificam suas coletas por causa de fatores exógenos como a quantidade de competidores nas flores, a distância entre as plantas e os ninhos, comunicação entre as campeiras, predadores, fatores climatológicos, entre outros, somado a fatores endógenos como a morfologia das peças florais, quantidade de néctar e sua concentração de açúcar, características do pólen e preferências especificas de cada visitante, entre outros, que podem facilitar ou dificultar as coletas de pólen e néctar obrigando essas abelhas a buscarem outras fontes até então não exploradas (Neff \& Simpson 1993; Hilário et al. 2001).

$\mathrm{Na}$ Amazônia, as abelhas nativas sem ferrão não fogem a regra e coletam o pólen e o néctar de plantas superiores, o endocarpo carnoso de Hymenaea sp. e, ocasionalmente, esporos de fungos (Absy \& Kerr 1977). Algumas abelhas do gênero Partamona, além do pólen e néctar, ampliaram a sua dieta e coletam outras partes da planta como pedaços do pedúnculo do caju (Anacardium occidentale L.).

Mesmo plantas com pólen não exposto protegidos em estames fechados, como os de espécies com anteras poricidas, são visitadas por Trigona que desenvolveram estratégias de coletas, que é de cortar as peças florais, e assim terem acesso ao alimento (Renner 1983). Já as abelhas Trigona williana Friese 1900, Trigona branneri Cockerell 1912 e Trigona fulviventris guianae Cockerell 1910 coletam o pólen caído no solo das inflorescências da palmeira Bactris gasipaes H.B.K. (Marques-Souza et al. 1996).

Apesar de seu hábito alimentar diversificado, até o presente não se tinham registros de que meliponíneos na Amazônia coletam o pólen de gimnosperma. A presença do pólen de Podocarpus sp. nas coletas de Frieseomelitta varia Lepeletier 1836 comprova que esses insetos não perdem uma oportunidade de explorar novas fontes e com isso aumentar

Instituto Nacional de Pesquisas da Amazônia, Coordenação de Botânica, Manaus, AM, Brasil

2 Autor para correspondência: msouza@inpa.gov.br 
suas opções quando da escassez de alimento, muito comum nessa região nos períodos chuvosos.

A Frieseomelitta varia é um meliponíneo neotropical com distribuição geográfica que vai do sudoeste Mexicano ao sudeste Brasileiro, sendo encontrado em florestas da Amazônia, em vegetação de caatinga e cerrado (MarquesSouza et al 1995; Teixeira et al. 2007). Ele não difere dos demais meliponíneos quanto aos hábitos de coletas, visitando muitas espécies vegetais, mas forrageando em um número reduzido de plantas.

Com o objetivo de contribuir com a biologia de meliponíneos na Amazônia, este trabalhoapresenta o primeiro relato da presença de pólen de Podocarpus sp. (Podocarpaceae) nas coletas de $F$. varia. Os hábitos de coletas desse inseto e os tipos polínicos aqui obtidos poderão ser utilizados para estimativas de época de floração de espécies vegetais e mesmo como parte de um calendário apícola mais geral dessa região.

\section{Material e métodos}

Os ninhos de Frieseomelitta varia foram introduzidos no Campus do INPA-Instituto Nacional de Pesquisas da Amazônia, em Manaus (AM), Brasil, ( $3^{\circ} 08^{\prime}$ 'S e a $60^{\circ} 10^{\prime} \mathrm{W}$ a uma altitude de 40.33 metros), uma área de $255.736,49 \mathrm{~m}^{2}$ (25,57ha), dentro do perímetro urbano da cidade, separada por duas grandes alamedas e ruas adjacentes, com intenso movimento de carros e cercada por residências.

A vegetação da floresta do Campus do INPA representa um estádio de regeneração de uma vegetação que foi cortada mas não queimada. Trata-se de uma floresta secundária com muitas espécies de floresta primária (Prance 1975; Gentry 1978).

O período estabelecido para a obtenção das amostras foi de agosto de 1995 a julho de 1996, em dias intercalados, sempre no horário entre 07:00 e 09:00h da manhã. As amostras de pólen foram obtidas de cinco abelhas de um ninho de Frieseomelitta varia que possuíam carregamento de pólen. Para isso, foi fechada a entrada da colméia e, aleatoriamente, capturadas essas abelhas. As suas cargas de pólen foram retiradas com o auxílio de um estilete e acondicionadas em vidros esterilizados. As abelhas foram libertadas após a retirada dos carregamentos de pólen de suas corbículas.

Em cada amostra de pólen foram adicionados $10 \mathrm{ml}$ de ácido acético glacial que permaneceram em repouso durante $24 \mathrm{~h}$. Em seguida, essas amostras foram acetolisadas pelo método de Erdtman (1960), montadas em lâminas com gelatina glicerinada e lutadas com parafina. Para cada amostra retirada das cinco abelhas foram feitas duas lâminas de pólen.

A identificação dos tipos polínicos foi feita por comparação com as lâminas de referência da Palinoteca do INPA-Instituto Nacional de Pesquisas da Amazônia e consultando-se a literatura especializada sobre o assunto.

De cada amostra foram contados em torno de 1.000 grãos de pólen e os resultados dessa contagem foram expressos em porcentagens. Esta metodologia foi adaptada de Vergeron (1964) que recomenda em amostras de méis esse número para atribuir a origem floral do produto.

O termo atratividade foi usado para indicar o grau de frequência que cada tipo polínico aparece nas coletas de Frieseomelitta varia, quanto mais frequente mais atrativo.

\section{Resultados}

$\mathrm{Na}$ Tab. 1 estão resumidos os tipos polínicos que foram coletados por Frieseomelitta varia Lepeletier 1836 no período de agosto de 1995 a julho de 1996. No total das coletas de pólen, os dados mostraram que durante um ano as operárias visitaram 79 espécies de plantas pertencentes a 60 gêneros e 37 famílias. Na distribuição mensal das coletas de pólen o número de espécies de plantas visitadas por esse meliponíneo variou de 20 em setembro a sete em junho (Tab. 2).

Das famílias botânicas constatou-se que as espécies de Cecropiaceae foram as mais frequentes, pois seus grãos de pólen estiveram presentes na coleta desse meliponíneo em todos os meses do ano. Em seguida, por ordem de importância, vieram as espécies de Myrtaceae, Sapotaceae e Moraceae presentes em onze, oito e cinco meses, respectivamente. Devido à grande abundância e frequência de pólen nas amostras de Frieseomelitta varia, as espécies dessas quatro famílias: Cecropia sp., Myrcia amazonica DC, Pouteria macrophylla (A.DC) Eyma e Morus sp., foram as responsáveis pela parcela mais significativa das coletas durante o período observado. Essas espécies contribuíram com $71 \%$ do total de pólen coletado durante um ano e forneceram a maior parte da alimentação para esse meliponíneo.

Outras espécies de plantas exerceram grande influência nas coletas de Frieseomelitta varia e apresentaram altas taxas de atratividade em meses consecutivos e/ou alternados. Foi o caso de Miconia sp. (Melastomataceae) frequente em oito meses e que teve seu pólen coletado significativamente em três, tendo sua maior atratividade em abril com $14,42 \%$. A Shefflera morototoni (Aubl.) Drude (Araliaceae), que teve seu pólen coletado durante dois meses e que apresentou atratividade de 7,10\% em abril e 26,04\% em maio. A espécie Aparisthimium cordatum (Juss.) Baill. (Euphorbiaceae), teve seus grãos de pólen coletados durante quatro meses e que apresentou atratividade de $22,36 \%$ no mês de dezembro. Ainda com relação ao tempo, para Terminalia sp. (Combretaceae), foram registrados seis meses de coleta, com destaque para sua atratividade de $10,57 \%$ em julho.

Houve outras plantas visitadas por Frieseomelitta varia com atratividade significativa, mas com pouca frequência de meses. Destaque para as espécies Pouteria caimito (Ruiz \& Pav.) Radlk. (Sapotaceae) e Stryphnodendron guianense Benth. (Mimosaceae) que tiveram pólens coletados pelas operárias em junho com atratividade de $24,79 \%$ e $18,05 \%$, respectivamente. A espécie Miconia myrianthera Benth. (Melastomataceae) frequente em três meses, teve atratividade significativa em agosto de 17,35\%. A espécie Vitex sp. (Verbenaceae) frequente apenas em abril, apresentou atratividade de 17,11\%. A Lindackeria paludosa (Benth.) Gilg. (Flacourtiaceae) presente em dois meses, mas atrativa somente em abril com 7,83\%; a espécie Echinodorus sp. (Alismataceae) frequente em março e com atratividade de 7,79\%. E, a Tapirira guianensis Aubl. (Anacardiaceae) cuja coleta de seu pólen apresentou atratividade significativa em setembro, de 7,04\%.

Quanto aos pólens de Podocarpus sp. (Podocarpaceae) nas coletas de Frieseomelitta varia (Fig. 1-4), eles representaram 4,94\% de atratividade no mês de março. Na Amazônia, há o relato da ocorrência de três espécies desse gênero, restritas a localidades bem específicas, que são: Podocarpus rospigliossi Pilg. encontrada na Região do Rio Pacaas Novos, Estado de Rondônia, coōrdenadas geográficas $11^{\circ}$ 
Tabela 1. Tipos polínicos coletados por Frieseomelitta varia Lepeletier 1836 no período de agosto de 1995 a julho de 1996 e sua atratividade (expressa em \%).

\begin{tabular}{|c|c|c|c|c|c|c|c|c|c|c|c|c|}
\hline FAMÍLIA/ESPÉCIE & AGO & SET & OUT & NOV & DEZ & JAN & FEV & MAR & $\mathrm{ABR}$ & MAI & JUN & JUL \\
\hline \multicolumn{13}{|l|}{ ACANTHACEAE } \\
\hline Acanthaceae tipo & & & & 0,36 & & & & & & & & \\
\hline Sanchezia sp. & & & & & & & & & & & & 0,02 \\
\hline \multicolumn{13}{|l|}{ ALISMATACEAE } \\
\hline Echinodorus sp. & & & & & & & & 7,79 & & & & \\
\hline \multicolumn{13}{|l|}{ AMARANTHACEAE } \\
\hline Celosia sp. & & & 1,31 & & 0,20 & & & & & & & \\
\hline \multicolumn{13}{|l|}{ ANACARDIACEAE } \\
\hline Mangifera indica Blume & & & & & & & 0,39 & & & & & \\
\hline Spondias mombin L. & & & 1,81 & & & & & & & & & \\
\hline Tapirira guianensis Aubl. & & 7,04 & & & & & & & & & & \\
\hline Thyrsodium sp. & & 0,45 & & & & & & & & & & \\
\hline \multicolumn{13}{|l|}{ APOCYNACEAE } \\
\hline Aspidosperma sp. & & 1,57 & 0,21 & & & & & & & & & \\
\hline \multicolumn{13}{|l|}{ ARALIACEAE } \\
\hline Schefflera morototoni (Aubl.) Frodin & & & & & & & & & 7,10 & 26,04 & & \\
\hline \multicolumn{13}{|l|}{ ARECACEAE } \\
\hline Cocos sp. & & & & & 0,95 & 5,03 & & 0,07 & & & & \\
\hline Euterpe oleracea Engel & & & & & & & & 0,42 & & & & \\
\hline Euterpe precatoria Mart. & & & & & & & & & 0,12 & 0,01 & & \\
\hline Euterpe sp. & & & & & & & 2,48 & & & & & \\
\hline Iriartella setigera $\mathrm{H}$. Wendl. & 7,24 & & & & & & & & & & & \\
\hline Mauritia flexuosa L. & 0,17 & & & & & & & & & & & \\
\hline Maximiliana sp. & & & & & & & 0,03 & & & & & \\
\hline Oenocarpus sp. & & & & & 0,01 & 0,89 & 3,97 & & & & & \\
\hline \multicolumn{13}{|l|}{ BIGNONIACEAE } \\
\hline Memora sp. & & & & & 0,02 & & & & & & & \\
\hline Tabebuia serratifolia (Vahl) G. Nicholson & & & & & & & & 0,18 & & & & \\
\hline Tabebuia sp. & 0,01 & & & & & & & & & & & \\
\hline \multicolumn{13}{|l|}{ BIXACEAE } \\
\hline Bixa orellana $\mathrm{L}$. & & & & & 0,04 & 0,75 & & & & & & \\
\hline \multicolumn{13}{|l|}{ CAESALPINIACEAE } \\
\hline Cassia latifolia G. Mey & & & & & & & 0,04 & & & & & \\
\hline Cassia quiquangulata L.C. Rich. & & & 0,11 & & & & & & & & & \\
\hline Cassia sp. & & & & 1,70 & & & & & & & & \\
\hline Cynometra sp. & & & & & & & & & & 1,90 & & \\
\hline Mora sp. & & 1,21 & & & & & & & & & & \\
\hline \multicolumn{13}{|l|}{ CARICACEAE } \\
\hline Carica papaya $\mathrm{L}$. & & & 0,69 & & 2,41 & 2,30 & & & & & & \\
\hline \multicolumn{13}{|l|}{ CECROPIACEAE } \\
\hline Cecropia sp. & 54,57 & 57,25 & 81,15 & 67,42 & 13,06 & 82,03 & 69,51 & 59,08 & 9,85 & 63,89 & 0,02 & 52,73 \\
\hline \multicolumn{13}{|l|}{ COMBRETACEAE } \\
\hline Combretum sp. & & & & 1,38 & 1,56 & & & 1,35 & & & & \\
\hline Terminalia sp. & 3,54 & 1,18 & 0,83 & & & & & & 3,58 & 2,28 & & 10,57 \\
\hline
\end{tabular}


Tabela 1. Continuação.

\begin{tabular}{|c|c|c|c|c|c|c|c|c|c|c|c|c|}
\hline FAMÍLIA/ESPÉCIE & AGO & SET & OUT & NOV & DEZ & JAN & FEV & MAR & $\mathrm{ABR}$ & MAI & JUN & JUL \\
\hline \multicolumn{13}{|l|}{ CLUSIACEAE } \\
\hline Vismia sp. & & 2,12 & & & & & & & & & & \\
\hline \multicolumn{13}{|l|}{ CYPERACEAE } \\
\hline Cyperus sp. & & & & & & & & & & & & 0,60 \\
\hline \multicolumn{13}{|l|}{ EUPHORBIACEAE } \\
\hline Alchornea schomburgkii Klotzsch. & & & 4,40 & 1,29 & & & & & & & & \\
\hline Aparisthmium cordatum (Juss.) Baill. & & & 1,53 & 8,19 & 22,36 & 0,17 & & & & & & \\
\hline Croton matourensis Aubl. & 0,05 & & & & & 0,06 & 0,68 & 0,11 & & & & \\
\hline Euphorbiaceae Tipo & 0,01 & & & & & & & & & & & \\
\hline \multicolumn{13}{|l|}{ FABACEAE } \\
\hline Dalbergia sp. & & & & & & & 0,11 & & & & & \\
\hline Fabaceae tipo & & & & 1,23 & & & & & & & & \\
\hline Vataireopsis speciosa Ducke & & 1,27 & & & & & & & & & & \\
\hline Vatairea sp. & & & & & & & & 0,61 & & & & \\
\hline \multicolumn{13}{|l|}{ FLACOURTIACEAE } \\
\hline Lindackeria paludosa (Benth.) Gilg. & & & & 0,23 & & & & & 7,83 & & & \\
\hline \multicolumn{13}{|l|}{ ICACINACEAE } \\
\hline Poraqueiba sericea B. Gates & & & & & & & & & & & & 0,35 \\
\hline \multicolumn{13}{|l|}{ LYTHRACEAE } \\
\hline Physocalymma scaberrimum Pohl & & & 0,35 & 2,83 & & & & & & & & \\
\hline \multicolumn{13}{|l|}{ MALPIGHIACEAE } \\
\hline Bannisteriopsis parviflora (Niedenzu) B. Gates & & & & & & & & & & & & 0,37 \\
\hline Byrsonima sp. & & 0,19 & 0,02 & & & & & & & & & \\
\hline \multicolumn{13}{|l|}{ MELASTOMATACEAE } \\
\hline Miconia myrianthera Benth. & 17,35 & 0,01 & & & & & & 0,62 & & & & \\
\hline Miconia sp. & 1,34 & 0,02 & 0,66 & & 0,08 & & 3,55 & 9,47 & 14,42 & & 6,62 & \\
\hline \multicolumn{13}{|l|}{ MELIACEAE } \\
\hline Melia $\mathrm{sp}$ & & 0,02 & & & & & & & & & & \\
\hline \multicolumn{13}{|l|}{ MIMOSACEAE } \\
\hline Inga sp. & & & & & & 0,10 & & & & & & \\
\hline Leucaena sp. & & & & & 1,79 & & & & & & & \\
\hline Mimosa invisa Mart. Ex Colla & & & & & & & & & 2,81 & 0,09 & & \\
\hline Mimosa pudica L. & & & & & 0,15 & 2,54 & 0,01 & 9,69 & 0,01 & & & \\
\hline Pentaclethra sp. & & 0,26 & & & & & & & & & & \\
\hline Stryphnodendron guianense Benth. & & 0,01 & & & & & & & 0,31 & & 18,05 & \\
\hline \multicolumn{13}{|l|}{ MORACEAE } \\
\hline Brosimum sp. & & & & & & & & & & & & 16,00 \\
\hline Morus sp. & & & & 14,17 & 37,46 & & & & 0,59 & & 13,88 & \\
\hline \multicolumn{13}{|l|}{ MYRTACEAE } \\
\hline Myrcia amazonica DC. & 12,20 & 18,15 & 4,23 & & 12,93 & 6,02 & 0,24 & 0,26 & 32,34 & 4,84 & 0,10 & \\
\hline Syzygium jambolanum DC. & 1,26 & & & & & & & & & & & 1,88 \\
\hline \multicolumn{13}{|l|}{ OLACACEAE } \\
\hline Aptandra sp. & & 4,19 & & & & & & & & & & \\
\hline \multicolumn{13}{|l|}{ OXALIDACEAE } \\
\hline Averrhoa carambola $\mathrm{L}$. & & & & & & & 0,04 & & & & & \\
\hline
\end{tabular}


562 Marques-Souza: Ocorrência do pólen de Podocarpus sp. (Podocarpaceae) nas coletas de Frieseomelitta varia Lepeletier 1836...

Tabela 1. Continuação.

\begin{tabular}{|c|c|c|c|c|c|c|c|c|c|c|c|c|}
\hline FAMÍLIA/ESPÉCIE & AGO & SET & OUT & NOV & DEZ & JAN & FEV & MAR & $\mathrm{ABR}$ & MAI & JUN & JUL \\
\hline \multicolumn{13}{|l|}{ PIPERACEAE } \\
\hline Piperonia sp. & & & & & 0,37 & & & & & & & \\
\hline \multicolumn{13}{|l|}{ POACEAE } \\
\hline Poaceae tipo 1 & & 0,44 & 0,99 & 0,38 & 0,03 & 0,11 & & & & & & \\
\hline Poaceae tipo 2 & & & & & 0,27 & & & & & & & \\
\hline \multicolumn{13}{|l|}{ PODOCARPACEAE } \\
\hline Podocarpus sp. & & & & & & & & 4,94 & & & & \\
\hline \multicolumn{13}{|l|}{ PORTULACACEAE } \\
\hline Talinum racemosum Rohrb. & & 0,07 & & & & & & & & & & \\
\hline \multicolumn{13}{|l|}{ RUBIACEAE } \\
\hline Duroia sp. & & & & 0,16 & 0,21 & & & & & & & \\
\hline Genipa americana $\mathrm{L}$. & 0,13 & & 1,26 & & & & & & & & & \\
\hline Rubiaceae tipo 2 & & & & & & & & & & & & 1,63 \\
\hline \multicolumn{13}{|l|}{ SAPINDACEAE } \\
\hline Matayba sp. & & & & & & & & & & 0,09 & & \\
\hline \multicolumn{13}{|l|}{ SAPOTACEAE } \\
\hline Pouteria caimito (Ruiz \& Pav.) Radlk. & & & & & & & & & & & 24,79 & \\
\hline Pouteria macrophylla (A.DC) Eyma & & & & & & & 18,63 & 5,43 & 3,91 & 0,33 & 36,54 & 15,68 \\
\hline Pouteria sp. & 0,41 & 1,05 & & & & & & & & & & \\
\hline Sapotaceae tipo 3 & 1,72 & & & & & & & & & & & \\
\hline \multicolumn{13}{|l|}{ SOLANACEAE } \\
\hline Solanum caavurana Vell. & & & & & & & & & & 0,53 & & \\
\hline Solanum sp. & & & & & & & & & & & & 0,17 \\
\hline \multicolumn{13}{|l|}{ VERBENACEAE } \\
\hline Lantana camara $\mathrm{L}$. & & & & & 6,11 & & & & & & & \\
\hline Vitex sp. & & 3,51 & 0,20 & & & & & & 17,11 & & & \\
\hline \multicolumn{13}{|l|}{ INDETERMINADO } \\
\hline Tricolporado reticulado & & & 0,25 & 0,67 & & & 0,31 & & & & & \\
\hline TOTAL (\%) & 100 & 100 & 100 & 100 & 100 & 100 & 100 & 100 & 100 & 100 & 100 & 100 \\
\hline
\end{tabular}

Tabela 2. Distribuição mensal da coleta de pólen de Frieseomelitta varia Lepeletier 1836

\begin{tabular}{|c|c|c|c|c|}
\hline Ano & Mês & Espécies & Gêneros & Famílias \\
\hline \multirow[t]{5}{*}{1995} & Agosto & 14 & 11 & 9 \\
\hline & Setembro & 20 & 18 & 16 \\
\hline & Outubro & 17 & 15 & 15 \\
\hline & Novembro & 13 & 11 & 11 \\
\hline & Dezembro & 19 & 17 & 16 \\
\hline \multirow[t]{7}{*}{1996} & Janeiro & 11 & 10 & 8 \\
\hline & Fevereiro & 14 & 13 & 11 \\
\hline & Março & 14 & 13 & 12 \\
\hline & Abril & 13 & 12 & 11 \\
\hline & Maio & 10 & 10 & 10 \\
\hline & Junho & 7 & 6 & 6 \\
\hline & Julho & 11 & 11 & 11 \\
\hline
\end{tabular}



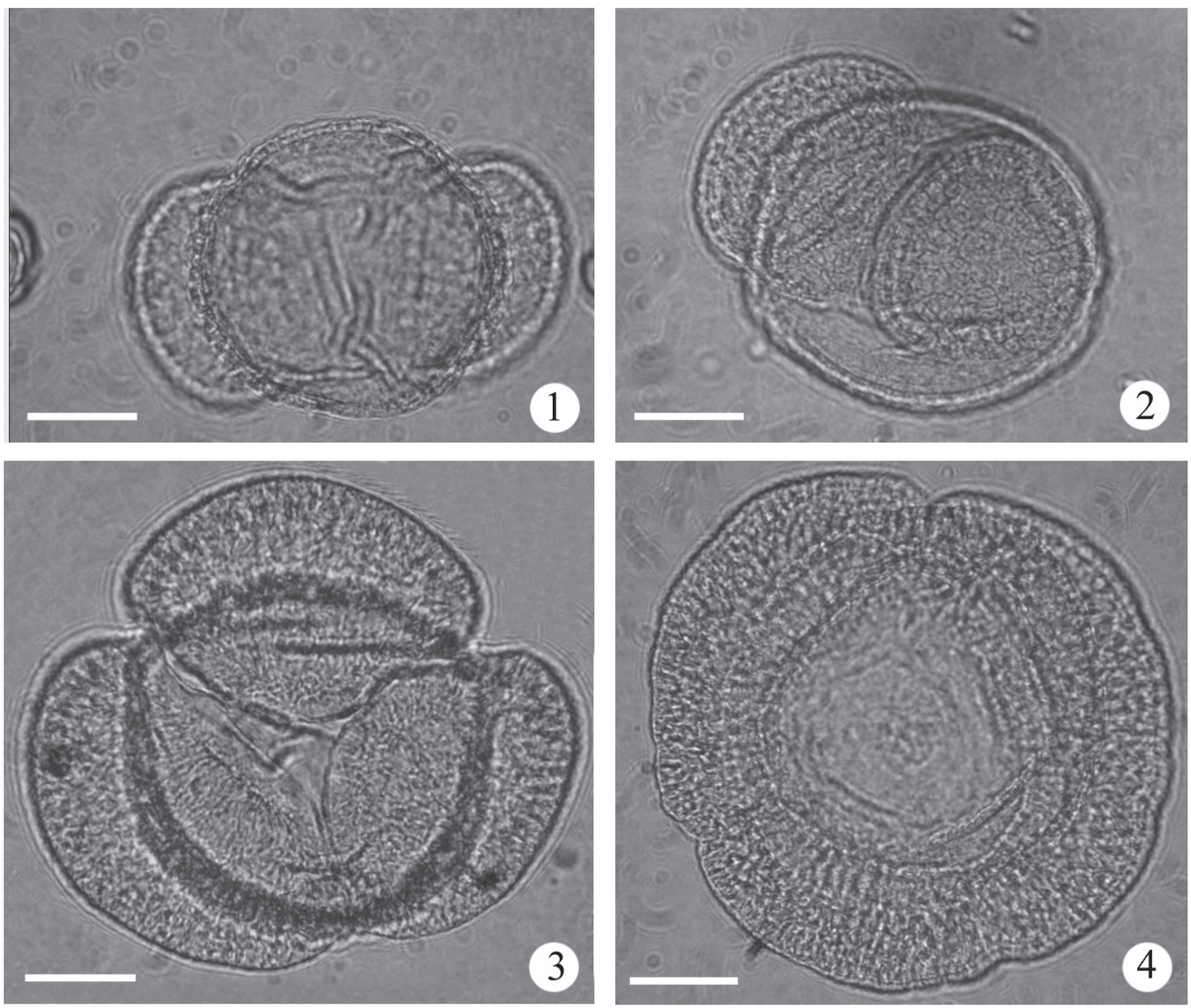

Figuras 1-2. Pólen de Podocarpus sp. mostrando os sacos laterais (vista equatorial). Figura 3. Pólen de Podocarpus sp. (vista distal). Figura 4. Pólen de Podocarpus sp. (vista polar). Escala $=20 \mu \mathrm{m}$.

$13^{\prime} \mathrm{Se} 63^{\circ} 35^{\prime} \mathrm{W}$ a $250-300 \mathrm{~m}$ de altitude; Podocarpus sellowii Klotzcch ex Endl. encontrada na Serra dos Pacaas Novos, Estado de Rondônia, coordenadas geográficas $10^{\circ} 52^{\prime} \mathrm{S}$ e $63^{\circ} 49^{\prime} \mathrm{W}$ a $1.000 \mathrm{~m}$ de altitude e Podocarpus roraimae Pilg. encontrada no Pico da Neblina, Estado do Amazonas, coordenadas geográficas $0^{\circ} 48^{\prime} \mathrm{S}$ e $66^{\circ} 2,41^{\prime} \mathrm{W}$ a $2.400 \mathrm{~m}$ de altitude (Fig. 5).

\section{Discussão}

Estudos de meliponíneos na Amazônia têm mostrado que abelhas consideradas como menos especializadas quanto à sua organização e comunicação, como Frieseomelitta varia, costumam se dispersar na procura pelo alimento e visitam plantas que são desprezadas pelas abelhas mais especializadas como as Melipona (Marques-Souza et al. 1996). Ao diversificar pouco em suas coletas de pólen e néctar, as Melipona aproveitam ao máximo as fontes mais atrativas e com isso mantêm certa fidelidade a determinadas espécies de plantas, fato que não ocorre com outros meliponíneos como Frieseomelitta sp. e Scaptotrigona sp. (MarquesSouza et al. 2002)

No presente estudo, observou-se que durante um ano, as operárias de Frieseomelitta varia diversificaram muito suas fontes de alimentos o que resultou na coleta do pólen de um número significativo de plantas. Absy \& Kerr (1977) que trabalharam na mesma área do presente estudo, constataram que operárias de Melipona seminigra merrillae Cockerell 1919 visitaram 33 espécies de plantas pertencentes a 33 gêneros e 21 famílias para coleta de pólen e 53 espécies de 33 gêneros pertencentes 21 famílias para coleta de néctar (Absy et al. 1980).

Outros estudos desenvolvidos na mesma área como o de Marques-Souza et al. (1995) constataram que operárias de Melipona rufiventris paraensis Ducke 1916 coletaram o pólen de 37 espécies de plantas distribuídas em 32 gêneros e 19 famílias e, em igual período, a Melipona compressipes manaosensis Schwarz 1932 coletou o pólen de 30 espécies de 


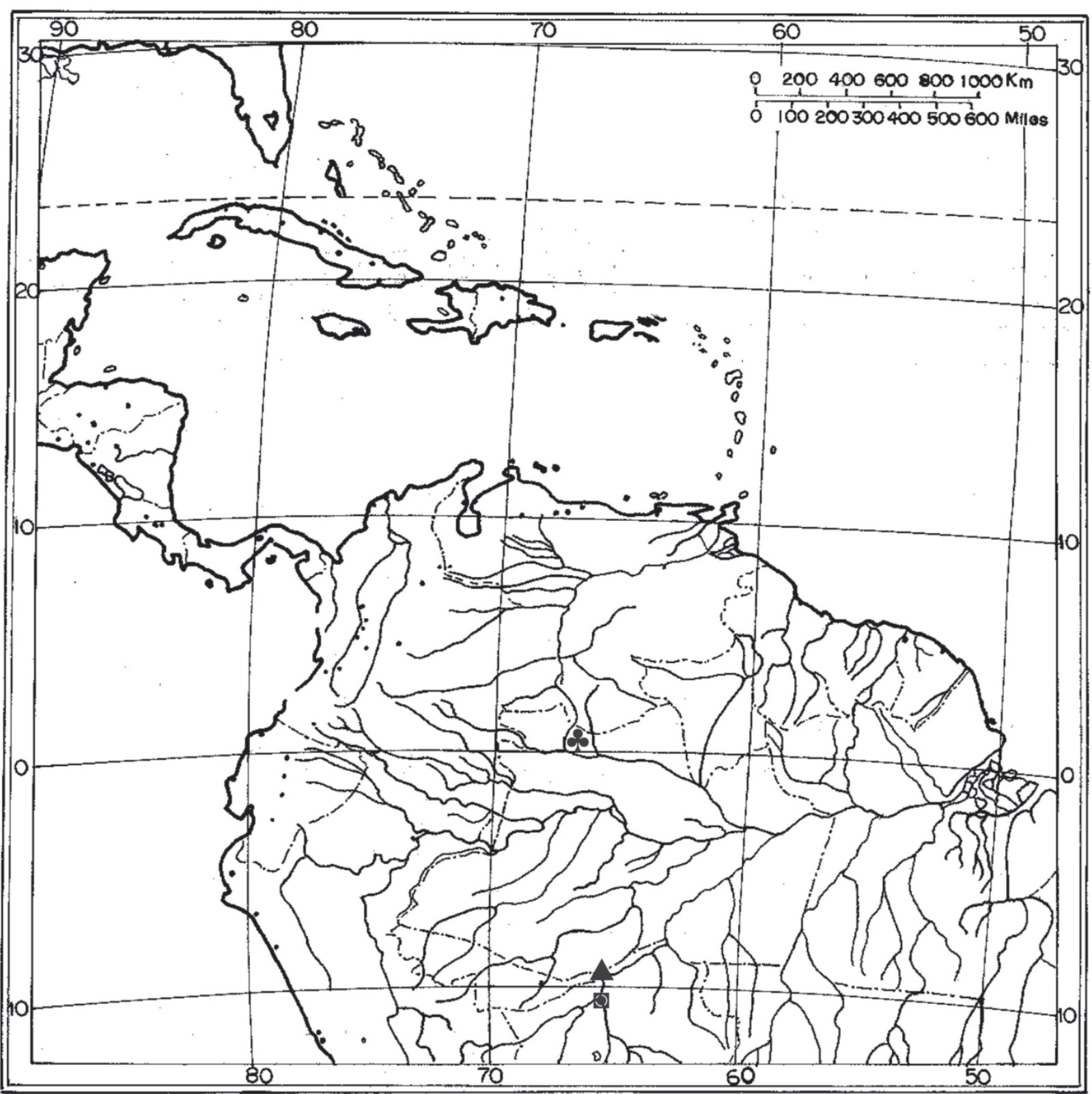

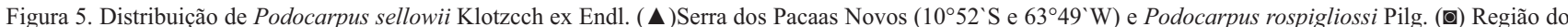

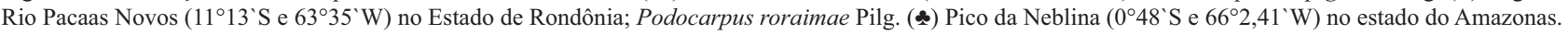

plantas distribuídas em 22 gêneros e 19 famílias (MarquesSouza 1996), enquanto que operárias de Trigona williana coletaram o pólen de 60 espécies de plantas distribuídas em 45 gêneros e 25 famílias (Marques-Souza et al. 1996).

Quando observados os números de espécies visitadas para a coleta de pólen e/ou néctar por meliponíneos na Amazônia, constata-se que somente um grupo reduzido de espécies fornece a maior parte do alimento que essas abelhas necessitam para se manterem. Mesmo nas coletas de abelhas tidas como menos especializadas há essa constatação. Das 79 espécies de plantas visitadas por Frieseomelitta varia, apenas quatro - Cecropia sp., Myrcia amazonica, Pouteria macrophylla e Morus sp. - forneceram $71 \%$ do total de pólen coletado, enquanto as outras 75 espécies de plantas responderam por $29 \%$ de suas coletas. Tal fato foi contatado por Marques-Souza et al. (2007), onde as operárias de Scaptotrigona fulvicutis Moure 1964 coletaram o pólen de 97 espécies de plantas sendo que apenas seis espécies foram as responsáveis pela parcela mais significativa da alimentação anual desse meliponíneo, correspondendo a $54,37 \%$ de todo o pólen coletado.

Esse número reduzidissimo de plantas que fornecem o grosso da alimentação dos meliponíneos é que determina a diferença do nicho trófico de cada espécie (MarquesSouza et al. 2002). Essas abelhas mantêm a fidelidade a um grupo pequeno de fontes alimentares, enquanto procuram 
diversificar as coletas do pólen para um número maior de plantas. Quando descobrem uma fonte em potencial tendem a explorar ao máximo os recursos ali contidos. Esse comportamento faz parte da estratégia dos meliponíneos de aproveitarem todas as oportunidades oferecidas, explorando as fontes com grande potencial, ao mesmo tempo em que diversificam e coletam em outras fontes menores. Com isso, há o aprendizado das abelhas na manipulação das peças florais dessas plantas que poderão ser incorporadas a sua dieta alimentar.

O aprendizado da manipulação das flores e de como utilizar com eficiência as fontes disponíveis são imprescindíveis para a sobrevivência das futuras gerações das abelhas. A pressão do meio é um dos fatores que influencia na evolução filogenética de vários gêneros e espécies de Apidae (Kerr 1969; Pessoti \& Le Sénéchal 1981).

Contudo, fontes que são intensamente coletadas por uma abelha podem ser desprezadas por outra. Mesmo o compartilhamento das fontes mais abundantes de pólen, aquelas com grandes floradas, não necessariamente são exploradas ao mesmo tempo. No caso da Frieseomelitta varia elas aproveitam para coletar o pólen remanescente da atividade das outras abelhas logo após o abandono das flores. Quando não, o compartilhamento do alimento se restringe em determinados ramos da planta.

A diversificação das coletas do pólen por espécies de meliponíneos na Amazônia não está diretamente relacionada com fatores abióticos como mudanças climáticas (Absy et al. 1984), e sim com outros fatores como disponibilidade do alimento, preferências específicas por determinadas plantas, época de floração e disputa pelas fontes mais vantajosas, onde as abelhas menores abandonam as flores quando da chegada das abelhas maiores, como as Melipona e Apis. Provavelmente, esse deslocamento constante das flores obrigou Frieseomelitta varia a diversificar a sua coleta para Podocarpus sp. (Podocarpaceae), uma gimnosperma que até o presente não se tinha conhecimento que era utilizada para pólen por meliponíneos em regiões neotropicais.

Como a família botânica Podocarpaceae não ocorre na área do presente estudo, tudo leva a crer que Frieseomelitta varia coletou o pólen de Podocarpus sp. em alguma residência, provavelmente introduzida em algum jardim, onde está sendo utilizada como planta ornamental. Até o presente não havia registros da distribuição dessa espécie fora das três áreas restritas da hiléia amazônica, duas em Rondônia e uma no Pico da Neblina no Amazonas. Van der Hammen \& Absy (1994) em seus estudos das mudanças climáticas na Amazônia durante o quaternário encontraram o pólen de Podocarpus sp. em sedimentos de lagos no Estado de Rondônia. Portanto, esse é o primeiro relato da presença do pólen de uma gimnosperma nas coletas de F. varia na Amazônia Central.

Pelos dados obtidos é possível afirmar que algumas espécies de plantas exerceram certa influência nas coletas de Frieseomelitta varia e apresentaram taxas de atratividade significativas em meses consecutivos e/ou alternados como Cecropia sp. (Cecropiaceae), Myrcia amazonica (Myrtaceae), Miconia sp. (Melastomataceae), Pouteria macrophylla (Sapotaceae) e a Terminalia sp. (Combretaceae) que possuem floração anual. Já as espécies Morus sp. (Moraceae), Miconia myrianthera (Melastomataceae), Shefflera morototoni (Araliaceae), Aparisthimium cordatum (Euphorbiaceae), Stryphnodendron guianense (Mimosaceae), Vitex sp. (Verbenaceae) e Lindackeria paludosa (Flacourtiaceae) são plantas estacionárias com períodos curtos de floração e a maioria só floresce no período seco que na Amazônia começa em julho (Tab. 1).

Cecropia sp. que foi intensamente visitada em doze meses por operárias de Frieseomelitta varia e cuja atratividade em janeiro foi de $82,03 \%$, é desprezada por muitas espécies de abelhas, enquanto as operárias de Apis mellifera L. e de Plebeia remota Holmberg 1903 coletam o pólen dessa planta (Ramalho et al. 1985; Cortopassi-Laurino \& Ramalho 1988; Marques-Souza et al. 1993).

As plantas estacionárias são muito importantes para as abelhas nativas da Amazônia, pois representam uma alternativa ao déficit na produção de pólen e néctar nos períodos de chuva, onde as abelhas chegam a ter dificuldades em se manterem (Gorenz 1967). Já as plantas com floração anual geralmente produzem muito pólen e com isso podem atender a todos os visitantes. É o caso de espécies de Myrcia (Myrtaceae) e de Miconia (Melastomataceae). As flores de Myrcia amazonica possuem densas inflorescências, com anteras rimosas o que expõe completamente o pólen facilitando a sua coleta pelos meliponíneos Numa comunidade de plantas onde diversos tipos de abelhas estão inseridos, é natural que algumas plantas sejam exploradas por todos. Mas, também, é natural que dentro dessa comunidade somente algumas delas sejam mais procuradas por um só tipo de abelha. São essas preferências alimentares de cada abelha, somado com a monopolização das fontes mais atrativas por um determinado visitante, que torna o nicho trófico de uma espécie diferente da outra (Absy et al. 1980; Simpson \& Neff 1981).

Muitas plantas visitadas por uma espécie de abelha, em muitos casos, não são as mais atrativas e sim representam alternativas de alimento. Mesmo quando uma abelha monopoliza por completo uma fonte alimentar, em certos casos marcando-a com feromônio, e passa a visitá-la com maior frequência, ainda assim, elas procuram outras alternativas em potencial. É comum quando capturados espécimes de Melipona na Amazônia os mesmos possuírem em seus carregamentos de pólen nas corbículas um, dois e até três tipos polínicos diferentes (Absy \& Kerr 1977). Quando quantificados comprova-se que há um tipo polínico dominante e os demais aparecem como pólen isolado. Nas amostras de Frieseomelitta varia, além desse perfil polínico, MarquesSouza et al. (1995) encontrou pólen acessório e concluiu que as melíponas aproveitavam melhor os recursos mais produtivos, enquanto operárias de $F$. varia se dispersavam à procura de outras fontes. 
Essa "dispersão" pode ser um problema de comunicação entre as campeiras e a colméia, que não conseguem passar às demais abelhas a localização correta das fontes em potencial. No período abrangido por este estudo foi verificado que os indivíduos de Frieseomelitta varia realizaram coletas diversificadas, pois coletaram o pólen de várias espécies de plantas. Absy \& Kerr (1977) observaram que a abelha Melipona seminigra merrillae Cockerell 1919 ao encontrar flores com boa recompensa alimentar, costumam explorar bem os recursos ali contidos, tirando o melhor proveito, evitando as fontes menos abundantes, que passam a ser exploradas quando as fontes mais vantajosas se esgotam.

Assim, é preciso observar quais outros fatores influenciam as coletas pelas abelhas. Não apenas a oferta de pólen/néctar de algumas espécies restrita a alguns períodos, ou aspectos relacionados à morfologia das suas flores que podem facilitar ou dificultar as coletas. O número de insetos competidores, a distância das fontes mais atrativas, a comunicação entre as abelhas, a presença de inimigos naturais, somado a diminuição sazonal do alimento, podem fazer com que essas abelhas busquem recursos até então nunca explorados, fato aqui exemplificado pela coleta de Podocarpus sp. por Frieseomelitta varia.

\section{Agradecimentos}

Ao CNPq (SWE-Processo 200038/96-6) por financiar parte dos estudos, a Cleonice de Oliveira Moura pela preparação das amostras de pólen e montagem das lâminas, ao Dr. Bruce Walker Nelson pela revisão do texto em inglês e ao Marcelo Maurício Duarte pelo tratamento das fotos e figura que ilustram este trabalho.

\section{Referências bibliográficas}

Referências bibliográficas

Absy, M.L. \& Kerr, W.E. 1977. Algumas plantas visitadas para a obtenção de pólen por operárias de Melipona seminigra merrillae em Manaus. Acta Amazonica 7(3): 309-315.

Absy, M.L.; Bezerra, E.B. \& Kerr, W.E. 1980. Espécies nectaríferas utilizadas por duas espécies de Melipona da Amazônia. Acta Amazonica 10(2): 271-281

Absy, M.L.; Camargo, J.M.F.; Kerr, W.E. \& Miranda, I.P.A. 1984. Espécies de plantas visitadas por Meliponinae (Hymenoptera; Apoidea), para coleta de pólen na região do médio Amazonas. Revista Brasileira de Biologia 44(2): 227-237.

Carvalho, C.A.L.; Moreti, A.C.C.; Marchini, L. C.; Alves, R.M.O. \& Oliveira, P.C.F. 2001. Pollen spectrum of "Uruçu" bee (Melipona scutellaris Latreille 1811). Revista Brasileira de Biologia 61(1): 63-67.

Cortopassi-Laurino, M. \& Ramalho, M. 1988. Pollen harvest by africanized Apis mellifera and Trigona spinipes in São Paulo botanical and ecological views. Apidologie 19: 1-24.

Erdtman, G. 1960. The acetolisys method in a revised description. Svensk Botanisk Tidskrift Lund 54: 561-564.

Gentry, A.H. 1978. Diversidade e regeneração da Capoeira do INPA, com referência especial às Bignoniaceae. Acta Amazonica 8(1): 67-70.

Gorenz, A.M. 1967. Períodos de secreção nectarífera na região de Belém, Pará. Atas do Simpósio sobre a Biota Amazonica 5: 203-206.

Guibu, L.S.; Ramalho M., Kleinert-Giovannini, A. \& Imperatriz-Fonseca, V.L. 1988. Exploração dos recursos florais por colônias de Melipona quadrifasciata (Apidae, Meliponinae). Revista Brasileira de Biologia 48(2): 299-305.

Hilário, S.D.; Imperatriz-Fonseca, V.L. \& Kleinert, A.M.P. 2001. Responses to climatic factors by foragers Plebeia pugnax Moure (in litt.) (Apidae, Meliponinae). Revista Brasileira de Biologia 61(2): 191-196.
Imperatriz-Fonseca, V.L.; Kleinert-Giovannini A. \& Ramalho M.. 1989. Pollen harvest by eusocial bees in a non-natural community in Brazil. Journal of Tropical Ecology 5: 239-242.

Kerr, W.E. 1969. Some aspects of the evolution of social bees. Evolutionary Biology 3(4): 119-175.

Kerr, W. E.; Absy, M. L. \& Marques-Souza, A. C. 1986/87. Espécies nectaríferas e poliníferas utilizadas pela abelha Melipona compressipes fasciculata (Meliponinae-Apidae) no Maranhão. Acta Amazonica 16-17( $\mathrm{n}^{\circ}$ único):145-156.

Kleinert-Giovannini, A. \& Imperatriz-Fonseca, V.L. 1987. Aspects of the trophic niche of Melipona marginata marginata Lepeletier (Apidae, Meliponinae). Apidologie 18: 69-100.

Marques-Souza, A.C.; Moura, C.O. \& Nelson, B.W. 1996. Pollen collected by Trigona williana (Hymenoptera: Apidae) in Central Amazonia. Revista de Biologia Tropical 44(2): 567-573.

Marques-Souza, A.C.; Absy, M.L.; Miranda, I.P.A. \& Küchmeister, H.E.C. 1993. Características de flores, néctar y visitantes de Kerianthera preclara (Rubiaceae). Revista de Biologia Tropical 41(3): 483-489.

Marques-Souza, A.C.; Absy, M.L.; Kerr, W.E. \& Aguilera-Peralta, F.J. 1995. Pólen coletado por duas espécies de meliponíneos (Hymenoptera: Apidae) da Amazônia. Revista Brasileira de Biologia 55(4): 855-864.

Marques-Souza, A.C. 1996. Fontes de pólen exploradas por Melipona compressipes manaosensis (Apidae: Meliponinae), abelha da Amazônia Central. Acta Amazonica 26(1/2): 77-86.

Marques-Souza, A.C.; Miranda, I.P.A.; Moura, C.O.; Rabelo, A. \& Barbosa, E.M. 2002. Características morfológicas e bioquímicas do pólen coletado por cinco espécies de meliponíneos da Amazônia Central. Acta Amazonica 32(2): 217-229.

Marques-Souza, A.C.; Absy, M.L. \& Kerr, W.E. 2007. Pollen harvest features of the Central Amazonian bee Scaptotrigona fulvicutis Moure 1964 (Apidae: Meliponinae), in Brazil. Acta Botanica Brasilica 21(1): 11-20.

Michener, C.D. 1979. Biogeography of the bees. Annals of the Missouri Botanical Garden 66(3): 277-347.

Neff, J.L. \& Simpson, B.B. 1993. Bees, pollination systems and plant diversity. Pp. 143-167. In: J. La Salle \& I.D. Gauld (eds.). Hymenoptera and Biodiversity. London, C.A.B. Internacional.

Pessoti, I. \& Le Sénéchal, A.M. 1981. Aprendizagem em abelhas. I-Discriminação simples em onze espécies. Acta Amazonica 11(3): 653-658.

Prance, G.T. 1975. The history of the INPA Capoeira based on ecological studies of Lecythidaceae. Acta Amazonica 5(3): 261-263.

Ramalho, M. 1990. Foraging by stingless bees of the genus, Scaptotrigona (Apidae, Meliponinae). Journal of Apicultural Research 29:(2) 61-67.

Ramalho, M.; Imperatriz-Fonseca, V.L.; Kleinert-Giovannini, A. \& Cortopassi-Laurino, M. 1985. Exploitation of floral resources by Plebeia remota Holmberg (Apidae, Meliponinae). Apidologie 16(3): 307-330.

Ramalho, M.; Giannini, T.C.; Malagodi-Braga, K.S. \& Imperatriz-Fonseca, V.L. 1994. Pollen harvest by stingless bee foragers (Hymenoptera, Apidae, Meliponinae). Grana 33: 239-244.

Ramalho, M.; Imperatriz-Fonseca, V.L. \& Giannini, T.C. 1998. Within colony size variation of foragers and pollen load capacity in the stingless bee Melipona quadrifasciata anthidioides Lepeletier (Apidae, Hymenoptera). Apidologie 29(3): 221-228.

Ramalho, M.; Silva, M.D. \& Carvalho, A.L. 2007. Dinâmica de uso das fontes de pólen por Melipona scutellaris Latreile (Hymenoptera, Apidae): uma análise comparativa com Apis mellifera (Hymenoptera, Apidae) no domínio tropical atlântico. Neotropical Entomology 36(1): 38-45

Renner, S. 1983. The widespread occurrence of anther destruction by Trigona bees in Melastomataceae. Biotropica 15(4): 251-256.

Simpson, B.B. \& Neff, J. L. 1981. Floral rewards: Alternatives to pollen and nectar. Annals of the Missouri Botanical Garden 68: 301-322.

Teixeira, A.F.R.; Oliveira, F.F. \& Viana, B.F. 2007. Utilization of floral resources by bees of the genus Frieseomelitta von Ihering (Hymenoptera: Apidae). Neotropical Entomology 36 (5): 675-684.

Van der Hammem, T. \& Absy, M.L. 1994. Amazonia during the last glacial. Palaeogeography, Palaeoclimatology, Palaeoecology 109: 247-261.

Vergeron, P. 1964. Interprétation statistique des résultats en matière d'analyse pollinique des miels. Annales de l'Abeille 7(4): 349-364

Versão eletrônica do artigo em www.scielo.br/abb e http://www.botanica.org.br/acta/ojs 